\title{
Second Hankel Determinant for Multivalent Spirallike and Convex Functions of Order $\alpha$
}

\author{
M. Elumalai ${ }^{1}$, C.Selvaraj ${ }^{2}$ \\ ${ }^{1}$ Department of Mathematics, Presidency College, Chennai, Tamil Nadu, India
}

\begin{abstract}
The objective of this paper is to obtain an upper bounded to the second Hankel determinant $\left|a_{p+1} a_{p+3}-a_{p+2}^{2}\right|$ for $\alpha-$ spiral starlike and convex $\alpha$-spiral function of $f$ and using Teoplitz determinants.
\end{abstract}

Keywords: Analytic functions, multivalent functions, $\alpha$-spiral starlike functions, convex $\alpha$-spiral function, upper bound, second Hankel determinant, positive real function, Toeplitz determinants.

\section{Introduction and Definitions}

Let $\mathrm{A}_{p}$ denote the class of functions analytic in $\mathrm{U}$ and having the power series expansion

$$
f(z)=z^{p}+\sum_{k=p+1}^{\infty} a_{k} z^{k} \quad(p \in \square=\{1,2,3, \ldots\})
$$

in the open unit disc $U=\{z:|z|<1\}$. Let $\mathrm{S}$ be the subclass of $A_{1}=A$, consisting of univalent functions.

In 1976, Noonan and Thomas defined the $q^{\text {th }}$ Hankel determinant of $f$ for $q \geq 1$ and $k \geq 1$ as

$$
H_{q}(k)=\left|\begin{array}{cccc}
a_{k} & a_{k+1} & \ldots & a_{k+q-1} \\
a_{k+1} & a_{k+2} & \ldots & a_{k+q} \\
\vdots & \vdots & \vdots & \vdots \\
a_{k+q-1} & a_{k+q} & \ldots & a_{k+2 q-2}
\end{array}\right| .
$$

This determinant has been considered by several authors in the literature. For example, Noonan and Thomas [21] studied about the second Hankel determinant of a really mean $p$ valent functions. Noor [22] determined the rate of growth of $H_{q}(k)$ as $k \rightarrow \infty$ for functions in $\mathrm{U}$ with bounded boundary rotation. Ehrenborg [8] considered the Hankel determinant of exponential polynomials. In [16], Layman considered Handel transform and obtained integrating properties.

Also, the Hankel determinant has been studied by various authors including Hayman [13] and Pommerenke [25]. We observe that $H_{2}(1)$ is nothing but the classical Fekete-Szegö functional. Then Fekete-Szegö further generalizes the estimate $\left|a_{3}-\mu a_{2}^{2}\right|$, where $\mu$ is real and $f \in \mathrm{U}$. Ali [3] finds sharp bounds on the first four coefficients and sharp estimate for the Fekete-Szegö functional $\gamma_{3}-t \gamma_{2}^{2}$, where $t$ is real. For our discussion in this paper, we consider the Hankel determinant for the case $q=2$ and $k=2$, known as second Hankel determinant,

$$
H_{2}(2)=\left|\begin{array}{ll}
a_{2} & a_{3} \\
a_{3} & a_{4}
\end{array}\right|=a_{2} a_{4}-a_{3}^{2} .
$$

Janteng, Halim and Darus [14] have determined the functional $\left|a_{2} a_{4}-a_{3}^{2}\right|$ and found a sharp bound for the functions $t$ in the subclass RT of $\mathrm{U}$, consisting of functions whose derivative has a positive real part, studied by Mac Gregor [17]. In this work, he has shown that if $f \in R T$, then $\left|a_{2} a_{4}-a_{3}^{2}\right| \leq \frac{4}{9}$. In [12], the authors obtained the second

Hankel determinant and sharp upper bounds for the familiar subclasses namely, starlike and convex functions denoted by ST and $\mathrm{CV}$ of $\mathrm{U}$ and have shown that $\left|a_{2} a_{4}-a_{3}^{2}\right| \leq 1$ and $\left|a_{2} a_{4}-a_{3}^{2}\right| \leq \frac{1}{8}$, respectively. Mishra and Gochhayat [18] have obtained sharp bound to the non-linear functional $\left|a_{2} a_{4}-a_{3}^{2}\right|$ for the class of analytic functions denoted by $R_{\lambda}(\alpha, \rho) \quad\left(0 \leq \rho \leq p, 0 \leq \lambda<p,|\alpha|<\frac{\pi}{2 p}\right)$. Similarly, the same coefficient inequality is calculated for certain subclasses of analytic functions by many authors, see e.g. [1], [4], [5], [1012], [18], [19], [25], [27-35].

Motivated by the earlier works obtained by different authors in this direction, we in the present paper, seek upper bound of the functional $\left|a_{2} a_{4}-a_{3}^{2}\right|$ for functions $t$ belonging to the classes $\operatorname{SP}_{p}(\alpha)$ and $C V S P_{p}(\alpha)$, defined as follows:

\section{Definition 1.1}

A function $f \in \mathrm{A}_{p}$ given by (1.1) is said to be $p$-valently $\alpha$-spiral if it satisfies the inequality

$$
\operatorname{Re} \frac{1}{p}\left\{e^{-i \alpha} \frac{z f^{\prime}(z)}{f(z)}\right\} \geq 0, \quad \forall(z \in \mathrm{U}), \quad|\alpha| \leq \frac{\pi}{2 p} .(1.4)
$$

We denote this class of functions by $S P_{p}(\alpha)$. Note that the class $S P_{p}(\alpha)$ reduces to $S P_{1}(\alpha)=: S P(\alpha)$, the class of $\alpha$-spiral functions introduced by spacek [30] and when $p=1$ and $\alpha=0$, it is ST, the class of starlike functions.

\section{Definition 1.2}

A function $f \in \mathrm{A}_{p}$ is said to be convex $\alpha$-spiral, where $\left(|\alpha| \leq \frac{\pi}{2 p}\right)$, if it satisfies the condition

$$
\operatorname{Re} \frac{1}{p}\left\{e^{-i \alpha}\left(1+\frac{z f^{\prime \prime}(z)}{f^{\prime}(z)}\right)\right\} \geq 0, \quad \forall z \in \mathrm{U} .
$$

The class of convex $\alpha$-spiral functions introduced by Rotertson is denoted by $\operatorname{CVSP}_{p}(\alpha)$.

It is observed when $\alpha=0, \operatorname{CVSP}_{p}(0)=C V$. 


\section{International Journal of Science and Research (IJSR) \\ ISSN (Online): 2319-7064}

Index Copernicus Value (2013): 6.14 | Impact Factor (2015): 6.391

Further, from the Definition 1.1 and 1.2, it is observed that, the Alexander type theorem [2] becomes true for the classes $S P_{p}(\alpha)$ and $C V S P_{p}(\alpha)$, stated as follow.

$$
f(z) \in \operatorname{CVSP}_{p}(\alpha) \text { if and only if } \frac{z f^{\prime}(z)}{p} \in S P_{p}(\alpha) .
$$

Some preliminary Lemmas needed for proving our results are as follows :

\section{Preliminary Results}

Let A be the family of all functions $h$ analytic in $\mathrm{U}$, for which $\operatorname{Re}\{h(z)\}>0$ and

$$
h(z)=1+\sum_{n=1}^{\infty} c_{n} z^{n}, \quad \forall z \in \mathrm{U} .
$$

\section{Lemma 2.1.}

[9] If $h \in \mathrm{A}$, then $\left|c_{k}\right| \leq 2$, for each $k \geq 1$.

\section{Lemma 2.2.}

[10] The power series for $h$ given in (2.1) converges in the unit disc $\mathrm{U}$ to a function in $\mathrm{A}$ if and only if the Toeplitz determinants.

$$
D_{k}=\left|\begin{array}{ccccc}
2 & c_{1} & c_{2} & \ldots & c_{k} \\
c_{-1} & 2 & c_{1} & \ldots & c_{k-1} \\
\vdots & \vdots & \vdots & \vdots & \\
c_{-k} & c_{-k+1} & c_{-k+2} & \ldots & 2
\end{array}\right|, \quad k=1,2,3, \ldots
$$

and $c_{-k}=\overline{c_{k}}$, are all non-negative. These are strictly positive except for $h(z)=\sum_{k=1}^{m} \rho_{k} h_{0} e^{i t_{k} z}, \rho_{k}>0, t_{k}$ real and $t_{k} \neq t_{j}$, for $k \neq j$, in this case $D_{k}>0$ for $k<(m-1)$ and $D_{k}=0$ for $k \geq m$.

This necessary and sufficient condition due to Carathéodory and Toeplitz can be found in [10]. We may assume without restriction that $c_{1}>0$ and on using Lemma 2.2, for $k=2$ and $k=3$ respectively, we get

$$
D_{2}=\left|\begin{array}{ccc}
2 & c_{1} & c_{2} \\
\overline{c_{1}} & 2 & c_{1} \\
\overline{c_{2}} & \overline{c_{1}} & 2
\end{array}\right|=\left[8+2 \operatorname{Re}\left\{c_{1}^{2} c_{2}\right\}-2\left|c_{2}\right|^{2}-4 c_{1}^{2}\right] \geq 0,
$$

which is equivalent to

$$
2 c_{2}=\left\{c_{1}^{2}+x\left(4-c_{1}^{2}\right)\right\}, \quad \text { for some } x, \quad|x| \leq 1 .
$$

$$
D_{3}=\left|\begin{array}{cccc}
2 & c_{1} & c_{2} & c_{3} \\
\overline{c_{1}} & 2 & c_{1} & c_{2} \\
\overline{c_{2}} & \overline{c_{1}} & 2 & c_{1} \\
\overline{c_{3}} & \frac{\bar{c}}{c_{2}} & \overline{c_{1}} & 2
\end{array}\right| \text {. }
$$

Then $D_{3} \geq 0$ is equivalent to

$$
\begin{array}{r}
\left(4 c_{3}-4 c_{1} c_{2}+c_{1}^{3}\right)\left(4-c_{1}^{2}\right)+c_{1}\left(2 c_{2}-c_{1}^{2}\right)^{2} \\
\leq 2\left(4-c_{1}^{2}\right)^{2}-2\left|2 c_{2}-c_{1}^{2}\right|^{2} .
\end{array}
$$

From the relations (2.2) and (2.3), after simplifying, we get

$$
\begin{aligned}
4 c_{3}=c_{1}^{3}+2 c_{1}\left(4-c_{1}^{2}\right) & x-c_{1}\left(4-c_{1}^{2}\right) x^{2} \\
+ & 2\left(4-c_{1}^{2}\right)\left(1-|x|^{2}\right) z
\end{aligned}
$$

for some real value of $x$, with $|x| \leq 1$.

\section{Main Result}

\section{Theorem 3.1.}

If $f(z)=z^{p}+\sum_{k=p+1}^{\infty} a_{k} z^{k} \in S P_{p}(\alpha)\left(-\frac{\pi}{2 p} \leq \alpha \leq \frac{\pi}{2 p}\right)$, then $\left|a_{p+1} a_{p+3}-a_{p+2}^{2}\right| \leq p^{2} \cos ^{2} \alpha$.

Proof:

Since $f(z)=z^{p}+\sum_{k=p+1}^{\infty} a_{k} z^{k} \in S P_{p}(\alpha)$, from the definition (1.1), there exists an analytic function $h \in \mathrm{A}$ in the unit disk U with $h(0)=1$ and $\operatorname{Re}\{h(z)\}>0$ such that

$$
\begin{aligned}
e^{-i \alpha}\left\{\frac{z f^{\prime}(z)}{p f(z)}\right\} & =h(z) \Rightarrow\left\{e^{-i \alpha} z f^{\prime}(z)+\mathrm{i} p \sin \alpha f(z)\right\} \\
& =p \cos \alpha\{f(z) \times h(z)\} .
\end{aligned}
$$

Replacing $f(z), f^{\prime}(z)$ by their equivalent $\mathrm{p}$-valent expressions and also the equivalent expression for $h(z)$ in series in (3.1), we have

$$
\begin{aligned}
e^{-i \alpha} z\left\{p z^{p-1}+\sum_{k=p+1}^{\infty} k a_{k} z^{k-1}\right\}+\mathrm{i} p \sin \alpha\left\{z^{p}+\sum_{k=p+1}^{\infty} a_{k} z^{k}\right\} \\
=p \cos \alpha\left[\left\{z^{p}+\sum_{k=p+1}^{\infty} a_{k} z^{k}\right\} \times\left\{1+\sum_{n=1}^{\infty} c_{n} z^{n}\right\}\right] .
\end{aligned}
$$

Upon simplification, we obtain

$$
\begin{aligned}
& e^{-i \alpha}\left(a_{p+1} z^{p}+2 a_{p+2} z^{p+1}+\ldots\right)=p \cos \alpha\left(c_{1} z^{p}\right. \\
& \left.\quad+\left(c_{2}+c_{1} a_{p+1}\right) z^{p+1}+\left(c_{3}+c_{2} a_{p+1}+c_{1} a_{p+2}\right) z^{p+2}+\ldots\right) .
\end{aligned}
$$

Equating the coefficients of like powers of $z^{p}, z^{p+1}$ and $z^{p+2}$ respectively in (3.2), we have

$$
\begin{gathered}
a_{p+1} e^{-i \alpha}=c_{1} p \cos \alpha, \\
2 a_{p+2} e^{-i \alpha}=\left(c_{2}+c_{1} a_{p+1}\right) p \cos \alpha \\
3 a_{p+3} e^{-i \alpha}=\left(c_{3}+c_{2} a_{p+1}+c_{1} a_{p+2}\right) p \cos \alpha .
\end{gathered}
$$

After simplifying, we get

$$
\begin{gathered}
a_{p+1}=e^{i \alpha} c_{1} p \cos \alpha, \\
a_{p+2}=\frac{e^{i \alpha}}{2}\left(c_{2}+c_{1}^{2} e^{i \alpha} p \cos \alpha\right) p \cos \alpha \\
a_{p+3}=\frac{e^{i \alpha}}{6}\left(c_{3}+3 c_{1} c_{2} e^{i \alpha} p \cos \alpha+c_{1}^{3} e^{i \alpha} p^{2} \cos ^{2} \alpha\right) p \cos \alpha .
\end{gathered}
$$

Substituting the values of $a_{p+1}, a_{p+2}$ and $a_{p+3}$ from (3.3) in the second Hankel functional $\left|a_{p+1} a_{p+3}-a_{p+2}^{2}\right|$ for the function $f \in \operatorname{SP}_{p}(\alpha)$, we have

$$
\begin{aligned}
&\left|a_{p+1} a_{p+3}-a_{p+2}^{2}\right|=\mid e^{i \alpha} c_{1} p \cos \alpha \\
& \times \frac{e^{i \alpha}}{6}\left\{2 c_{3}+3 c_{1} c_{2} e^{i \alpha} p \cos \alpha+c_{1}^{3} e^{2 i \alpha} p \cos ^{2} \alpha\right\} p \cos \alpha \\
& \quad-\frac{e^{2 i \alpha}}{4}\left\{c_{2}+c_{1}^{2} e^{i \alpha} p \cos \alpha\right\}^{2} p^{2} \cos ^{2} \alpha \mid .
\end{aligned}
$$

Using the facts $|x a+y b| \leq|x||a|+|y||b|$, where $x, y, a$ and $b$ are real numbers and $\left|e^{\text {ni }}\right|=1$, upon simplification, we obtain

$$
\left|a_{p+1} a_{p+3}-a_{p+2}^{2}\right| \leq \frac{p^{2} \cos ^{2} \alpha}{12} \times\left|4 c_{1} c_{3}-3 c_{2}^{2}-c_{1}^{4} p^{2} \cos ^{2} \alpha\right| .
$$

Substituting the values of $c_{2}$ and $c_{3}$ from (2.2) and (2.4) respectively, we have 


\section{International Journal of Science and Research (IJSR) \\ ISSN (Online): 2319-7064}

Index Copernicus Value (2013): 6.14 | Impact Factor (2015): 6.391

$$
\begin{gathered}
\left|4 c_{1} c_{3}-3 c_{2}^{2}-c_{1}^{4} p^{2} \cos ^{2} \alpha\right|=\mid 4 c_{1} \\
\times \frac{1}{4}\left\{c_{1}^{3}+2 c_{1}\left(4-c_{1}^{2}\right) x-c_{1}\left(4-c_{1}^{2}\right) x^{2}+2\left(4-c_{1}^{2}\right)\left(1-|x|^{2}\right) z\right\} \\
-3 \times \frac{1}{4}\left\{c_{1}^{2}+x\left(4-c_{1}^{2}\right)\right\}^{2}-c_{1}^{4} p^{2} \cos ^{2} \alpha \mid .
\end{gathered}
$$

Using the fact $|x| \leq 1$, upon simplification, we obtain $4\left|4 c_{1} c_{3}-3 c_{2}^{2}-c_{1}^{4} p^{2} \cos ^{2} \alpha\right| \leq \mid\left(1-4 p^{2} \cos ^{2} \alpha\right) c_{1}^{4}+8 c_{1}\left(4-c_{1}^{2}\right)$

$$
+2 c_{1}^{2}\left(4-c_{1}^{2}\right)|x|-\left(c_{1}+2\right)\left(c_{1}+6\right)\left(4-c_{1}^{2}\right)|x|^{2} \mid .
$$

Since $c_{1} \in[0,2]$, using the result $\left(c_{1}+a\right)\left(c_{1}+b\right) \geq\left(c_{1}-a\right)\left(c_{1}-b\right)$, where $a, b \geq 0$ on the right-hand side of the above inequality we get

$$
\begin{aligned}
& 4\left|4 c_{1} c_{3}-3 c_{2}^{2}-c_{1}^{4} p^{2} \cos ^{2} \alpha\right| \leq \mid\left(1-4 p^{2} \cos ^{2} \alpha\right) c_{1}^{4} \\
& +8 c_{1}\left(4-c_{1}^{2}\right)+2 c_{1}^{2}\left(4-c_{1}^{2}\right)|x|-\left(c_{1}-2\right)\left(c_{1}-6\right)\left(4-c_{1}^{2}\right)|x|^{2} \mid .
\end{aligned}
$$

Choosing $c_{1}=c, c \in[0,2]$, applying triangle inequality and replacing $|x|$ by $\delta$ on the right hand side of (3.5) we obtain $4\left|4 c_{1} c_{3}-3 c_{2}^{2}-c_{1}^{4} p^{2} \cos ^{2} \alpha\right| \leq \mid\left(4 p^{2} \cos ^{2} \alpha-1\right) c_{1}^{4}+8 c_{1}\left(4-c_{1}^{2}\right)$

$$
+2 c_{1}\left(4-c_{1}^{2}\right) \delta+\left(c_{1}-2\right)\left(c_{1}-6\right)\left(4-c_{1}^{2}\right) \delta^{2} \mid .
$$$$
=F(c, \delta) \text {, with } 0 \leq \delta=|x| \leq 1 \text {, }
$$

where

$$
\begin{array}{r}
F(c, \delta)=\left(4 p^{2} \cos ^{2} \alpha-1\right) c_{1}^{4}+8 c_{1}\left(4-c_{1}^{2}\right)+2 c_{1}\left(4-c_{1}^{2}\right) \delta \\
+\left(c_{1}-2\right)\left(c_{1}-6\right)\left(4-c_{1}^{2}\right) \delta^{2} .
\end{array}
$$

Now the function $F(c, \delta)$ is maximized on the closed square $[0,2] \times[0,1]$. Differentiating $F(c, \delta)$ in $(3.7)$, partially with respect to $\delta$, we get

$$
\frac{\partial F}{\partial \delta}=2\left[c^{2}+(c-2)(c-6) \delta\right]\left(4-c^{2}\right)
$$

for $0 \leq \delta \leq 1$, for fixed c with $0 \leq c \leq 2$, from (3.8) we observe that $\frac{\partial F}{\partial \delta}>0$.

Consequently, $F(c, \delta)$ is an increasing function of $\delta$ and hence cannot have maximum value at any point in the interior of the closed square $[0,2] \times[0,1]$. Moreover, for fixed $c \in[0,2]$, we have

$$
\max _{0 \leq \delta \leq 1} F(c, \delta)=F(c, 1)=G(c) .
$$

Upon simplifying the relation (3.7) and (3.9) we obtain

$$
G(c)=4\left(p^{2} \cos ^{2} \alpha-1\right) c^{4}+48 \text {. }
$$

Differentiation yields:

$$
G^{\prime}(c)=16\left(p^{2} \cos ^{2} \alpha-1\right) c^{3}
$$

From the expression (3.11), we observe that $G^{\prime}(c) \leq 0$ from all values of $c$ in the interval $0 \leq c \leq 2$ and for a fixed valued of $\alpha$ with $\left(-\frac{\pi}{2 p} \leq \alpha \leq \frac{\pi}{2 p}\right)$. Therefore, $G(c)$ is a monotonically decreasing function of $c$ in the interval $[0,2]$. So, that its maximum value occurs at $c=0$. From (3.10), we get

$$
\max _{0 \leq c \leq 2} G(0)=48 \text {. }
$$

After simplifying the expressions (3.6) and (3.12) we obtain $\left|4 c_{1} c_{3}-3 c_{2}^{2}-c_{1}^{4} p^{2} \cos ^{2} \alpha\right| \leq 12$.

Upon simplifying the expressions (3.4) and (3.13), we get

$$
\left|a_{p+1} a_{p+3}-a_{p+2}^{2}\right| \leq p^{2} \cos ^{2} \alpha .
$$

Choosing $c_{1}=c=0$ and selecting $x=-1$ in (2.2) and (2.4), we find that $c_{2}=-2$ and $c_{3}=0$. Substituting these values in
(3.13), it is observed that equality is attained which shows that our result is sharp. This completes the proof of our Theorem 3.1.

Choosing $p=1$ from (3.14) following

\section{Corollary 3.2.}

[37] If $f(z) \in S P(\alpha)$, then $-\frac{\pi}{2} \leq \alpha \leq \frac{\pi}{2}$ $\left|a_{2} a_{4}-a_{3}^{2}\right| \leq \cos ^{2} \alpha$.

For the choice of $p=1$ and $\alpha=0$ from (3.14) following

\section{Corollary 3.3.}

If $f(z) \in S P(\alpha)$, then

$\left|a_{2} a_{4}-a_{3}^{2}\right| \leq 1$.

This inequality is sharp and concides with that of Janteng, Halim and Darus [14].

\section{Theorem 3.4.}

If $f(z) \in \operatorname{CVSP}_{p}(\alpha) \quad\left(|\alpha| \leq \frac{\pi}{2 p}\right)$, then

$$
\begin{aligned}
& \left|a_{p+1} a_{p+3}-a_{p+2}^{2}\right| \\
& \leq \frac{p^{4}\left\{\begin{array}{c}
6\left(1+2 p \cos \alpha+p^{2} \cos ^{2} \alpha\right)+(p+1)(p+3) \\
\left(p^{2}+4 p+7+2\left(p^{2}+4 p+1\right) p^{2} \cos ^{2} \alpha\right)
\end{array}\right\}}{(p+1)(p+2)^{2}(p+3)\left\{\begin{array}{c}
2\left(p^{2}+4 p+1\right) \\
+\left(p^{2}+4 p+7\right) p^{2} \sec ^{2} \alpha
\end{array}\right\}} .
\end{aligned}
$$

Proof:

Since $f(z)=z^{p}+\sum_{k=p+1}^{\infty} a_{k} z^{k} \in \operatorname{CVSP}_{p}(\alpha)$, from the definition (1.2), there exists an analytic function $h \in \mathrm{A}$ in the unit disk U with $h(0)=1$ and $\operatorname{Re}\{h(z)\}>0$ such that

$$
\begin{aligned}
\frac{1}{p}\left[e^{-i \alpha}\left\{1+\frac{z f^{\prime \prime}(z)}{f^{\prime}(z)}\right\}\right] & =h(z) \Leftrightarrow\left\{e^{-i \alpha}\left\{f^{\prime}(z)+z f^{\prime \prime}(z)\right\}+i p \sin \alpha f^{\prime}(z)\right\} \\
& =p \cos \alpha\left\{f^{\prime}(z) \times h(z)\right\}
\end{aligned}
$$

Replacing $f^{\prime}(z), f^{\prime \prime}(z)$ and $h(z)$ with their equivalent series expressions in (3.1), we have

$$
\begin{gathered}
e^{-i \alpha}\left\{p z^{p-1}+\sum_{k=p+1}^{\infty} k a_{k} z^{k-1}\right\}+z\left\{p(p-1) z^{p-2}+\sum_{k=p+1}^{\infty} k(k-1) a_{k} z^{k-2}\right\} \\
+i p \sin \alpha\left\{z+\sum_{k=2}^{\infty} a_{k} z^{k}\right\} \\
=p \cos \alpha\left[\left\{p z^{p-1}+\sum_{k=p+1}^{\infty} k a_{k} z^{k-1}\right\} \times\left\{1+\sum_{n=1}^{\infty} c_{n} z^{n}\right\}\right] .
\end{gathered}
$$

Upon simplification, we obtain

$$
\begin{aligned}
e^{-i \alpha}\left\{(p+1) a_{p+1} z^{p}+\right. & \left.\left.2(p+2) a_{p+2} z^{p+1}+3(p+3) a_{p+3} z^{p+2}+\ldots\right)\right\} \\
=p \cos \alpha[ & {\left[p c_{1} z^{p}+\left\{p c_{2}+(p+1) c_{1} a_{p+1}\right\} z^{p+1}\right.} \\
& \left.+\left\{p c_{3}+(p+1) c_{2} a_{p+1}+(p+2) c_{1} a_{p+2}\right\} z^{p+2}+\ldots\right] .
\end{aligned}
$$

Equating the coefficients of like powers of $z^{p}, z^{p+1}$ and $z^{p+2}$ respectively in (3.16), we have

$$
\begin{gathered}
(p+1) a_{p+1} e^{-i \alpha}=p c_{1} p \cos \alpha, \\
2(p+2) a_{p+2} e^{-i \alpha}=\left\{p c_{2}+(p+1) c_{1} a_{p+1}\right\} p \cos \alpha, \\
3(p+2) a_{p+3} e^{-i \alpha}=\left\{p c_{3}+(p+1) c_{2} a_{p+1}+(p+2) c_{1} a_{p+2}\right\} p \cos \alpha
\end{gathered}
$$

After simplifying, we get 
$a_{p+1}=\frac{e^{i \alpha}}{p+1} c_{1} p^{2} \cos \alpha$

$a_{p+2}=\frac{e^{i \alpha}}{2(p+2)}\left(c_{2}+c_{1}^{2} e^{i \alpha} p \cos \alpha\right) p^{2} \cos \alpha$

$a_{p+3}=\frac{e^{i \alpha}}{6(p+3)}\left(c_{3}+3 c_{1} c_{2} e^{i \alpha} p \cos \alpha+c_{1}^{3} e^{2 i \alpha} p^{2} \cos ^{2} \alpha\right) p^{2} \cos \alpha$

Substituting the values of $a_{p+1}, a_{p+2}$ and $a_{p+3}$ from (3.17) in the second Hankel functional $\left|a_{p+1} a_{p+3}-a_{p+2}^{2}\right|$ for the function $f \in C V S P_{p}(\alpha)$, applying the same procedure as described in Theorem

3.1, upon simplification, we obtain

$$
\begin{array}{r}
\left|a_{p+1} a_{p+3}-a_{p+2}^{2}\right|=\frac{p^{4} \cos ^{2} \alpha}{24(p+1)(p+2)^{2}(p+3)} \mid 8(p+2)^{2} c_{1} c_{3}+12 p c_{1}^{2} c_{2} \cos \alpha \\
-6(p+1)(p+3) c_{2}^{2}-\left(p^{2}+4 p+7\right) 2 p^{2} c_{1}^{4} \cos ^{2} \alpha \mid .
\end{array}
$$

The above expression is equivalent to

$$
\begin{aligned}
&\left|a_{p+1} a_{p+3}-a_{p+2}^{2}\right|= \frac{p^{4} \cos ^{2} \alpha}{12(p+1)(p+2)^{2}(p+3)} \\
& \times\left|d_{1} c_{1} c_{3}+d_{2} c_{1}^{2} c_{2}+d_{3} c_{2}^{2}+d_{4} c_{1}^{4}\right|
\end{aligned}
$$

where

$d_{1}=4(p+2)^{2}, d_{2}=6 p \cos \alpha, d_{3}=-3(p+1)(p+3)=-3\left(p^{2}+4 p+3\right)$

$d_{4}=-\left(p^{2}+4 p+7\right) p^{2} \cos ^{2} \alpha$.

Substituting the values of $c_{2}$ and $c_{3}$ from (2.2) and (2.4) respectively from lemma 2.2 in the right hand side of (3.18), we have

$$
\begin{array}{r}
\left|d_{1} c_{1} c_{3}+d_{2} c_{1}^{2} c_{2}+d_{3} c_{2}^{2}+d_{4} c_{1}^{4}\right|=\mid d_{1} c_{1} \times \frac{1}{4}\left\{c_{1}^{3}+2 c_{1}\left(4-c_{1}^{2}\right) x-c_{1}\left(4-c_{1}^{2}\right) x^{2}\right. \\
\left.+2\left(4-c_{1}^{2}\right)\left(1-|x|^{2}\right) z\right\}+d_{2} c_{1}^{2} \times \frac{1}{2}\left\{c_{1}^{2}+x\left(4-c_{1}^{1}\right)\right\} \\
+d_{3} \times\left\{c_{1}^{2}+x\left(4-c_{1}^{2}\right)\right\}^{2}+d_{4} c_{1}^{4} \mid .
\end{array}
$$

After simplifying, we get

$$
\begin{array}{r}
4\left|d_{1} c_{1} c_{3}+d_{2} c_{1}^{2} c_{2}+d_{3} c_{2}^{2}+d_{4} c_{1}^{4}\right|=\mid\left(d_{1}+2 d_{2}+d_{3}+4 d_{4}\right) c_{1}^{4} \\
+2 d_{1} c_{1}\left(4-c_{1}^{2}\right) z+2 d_{1} c_{1}\left(4-c_{1}^{2}\right) z+2\left(d_{1}+d_{2}+d_{3}\right) c_{1}^{2}\left(4-c_{1}^{2}\right)|x| \\
+\left\{\left(d_{1}+d_{3}\right) c_{1}^{2}+2 d_{1} c_{1}-4 d_{3}\right\}\left(4-c_{1}^{2}\right)|x|^{2} z \mid
\end{array}
$$

Using the values of $d_{1}, d_{2}, d_{3}$ and $d_{4}$ from the relation (3.19), upon simplification, we obtain

$$
\begin{aligned}
& d_{1}+2 d_{2}+d_{3}+4 d_{4}=p^{2}+4 p+7+12 p \cos \alpha \\
& -4\left(p^{2}+4 p+1\right) p^{2} \cos ^{2} \alpha \\
& d_{1}=4(p+2)^{2} \\
& d_{1}+d_{2}+d_{3}=p^{2}+4 p+7-6 p \cos \alpha \text {. } \\
& \left(d_{1}+d_{3}\right) c_{1}^{2}+2 d_{1} c_{1}-4 d_{3}=\left(p^{2}+4 p+7\right) c_{1}^{2}-8(p+2)^{2} c_{1} \\
& +12(p+1)(p+3)\} \text {. } \\
& \left\{\left(p^{2}+4 p+7\right) c_{1}^{2}+8(p+2)^{2} c_{1}+12(p+1)(p+3)\right\}= \\
& \left(p^{2}+4 p+7\right) \times\left[c_{1}^{2}+\frac{8(p+2)^{2}}{\left(p^{2}+4 p+7\right)} c_{1}+\frac{12(p+1)(p+3)}{\left(p^{2}+4 p+7\right)}\right] \\
& =\left(p^{2}+4 p+7\right) \times\left[\left\{c_{1}+\frac{4(p+2)^{2}}{\left(p^{2}+4 p+7\right)}\right\}^{2}-\frac{16(p+2)^{4}}{\left(p^{2}+4 p+7\right)^{2}}+\frac{12(p+1)(p+3)}{\left(p^{2}+4 p+7\right)}\right]
\end{aligned}
$$

Upon simplification, the above expression can be expressed as

$$
\begin{gathered}
\left\{\left(p^{2}+4 p+7\right) c_{1}^{2}+8(p+2)^{2} c_{1}+12(p+1)(p+3)\right\}=\left(p^{2}+4 p+7\right) \\
\times\left[\left\{c_{1}+\frac{4(p+2)^{2}}{\left(p^{2}+4 p+7\right)}\right\}^{2}-\left\{\frac{2 \sqrt{p^{4}+8 p^{3}+18 p^{2}+8 p}+1}{\left(p^{2}+4 p+7\right)}\right\}^{2}\right] . \\
\left\{\left(p^{2}+4 p+1\right) c_{1}^{2}+8(p+2)^{2} c_{1}+12(p+1)(p+3)\right\}=\left(p^{2}+4 p+7\right) \\
\times\left[c_{1}+\left\{\frac{4(p+2)^{2}}{\left(p^{2}+4 p+7\right)}+\frac{2 \sqrt{p^{4}+8 p^{3}+18 p^{2}+8 p}+1}{\left(p^{2}+4 p+7\right)}\right\}\right] \\
\times\left[c_{1}+\left\{\frac{4(p+2)^{2}}{\left(p^{2}+4 p+7\right)}-\frac{2 \sqrt{p^{4}+8 p^{3}+18 p^{2}+8 p}+1}{\left(p^{2}+4 p+7\right)}\right\}\right] .
\end{gathered}
$$

Since $c_{1} \in[0,2]$, using the results

$\left(c_{1}+a\right)\left(c_{1}+b\right) \geq\left(c_{1}-a\right)\left(c_{1}-b\right)$, where $a, b \geq 0$ in the right hand side of (3.23)

$$
\begin{aligned}
& \left\{\left(p^{2}+4 p+1\right) c_{1}^{2}+8(p+2)^{2} c_{1}+12(p+1)(p+3)\right\} \\
& \quad \geq\left\{\left(p^{2}+4 p+1\right) c_{1}^{2}-8(p+2)^{2} c_{1}+12(p+1)(p+3)\right\} .
\end{aligned}
$$

From the relation (3.22) and (3.24), we obtain

$$
\begin{aligned}
&-\left\{\left(d_{1}+d_{3}\right) c_{1}^{2}+2 d_{1} c_{1}-4 d_{3}\right\} \geq-\left\{\left(p^{2}+4 p+1\right) c_{1}^{2}\right. \\
&\left.-8(p+2)^{2} c_{1}+12(p+1)(p+3)\right\} .
\end{aligned}
$$

Substituting the calculated values from (3.21) and (3.25) in the right hand side of the relation (3.20), we get

$4\left|d_{1} c_{1} c_{3}+d_{2} c_{1}^{2} c_{2}+d_{3} c_{2}^{2}+d_{4} c_{1}^{4}\right| \leq \mid\left\{p^{2}+4 p+7+12 p \cos \alpha\right.$

$\left.-4\left(p^{2}+4 p+1\right) p^{2} \cos ^{2} \alpha\right\} c_{1}^{4}+2\left(p^{2}+4 p+7+6 p \cos \alpha\right) c_{1}^{2}\left(4-c_{1}^{2}\right)|x|$

$-\left\{\left(p^{2}+4 p+7\right) c_{1}^{2}-8(p+2)^{2} c_{1}+12(p+1)(p+3)\right\}\left(4-c_{1}^{2}\right)|x|^{2} z \mid \cdot(3.26)$

Choosing $c_{1}=c \in[0,2]$, applying Triangle inequality and replacing $|x|$ by $\mu$ in the right hand side of (3.20), it reduces to

$=F(c, \delta)$, for $0 \leq \delta=|x| \leq 1$,

where

$$
\begin{aligned}
& F(c, \mu)=\left[\left\{p^{2}+4 p+7+12 p \cos \alpha-4\left(p^{2}+4 p+1\right) p^{2} \cos ^{2} \alpha\right\} c_{1}^{4}\right. \\
& +8(p+2)^{2} c_{1}\left(4-c_{1}^{2}\right) z+2\left(p^{2}+4 p+7+6 p \cos \alpha\right) c_{1}^{2}\left(4-c_{1}^{2}\right) \delta \\
& \left.+\left\{\left(p^{2}+4 p+7\right) c_{1}^{2}-8(p+2)^{2} c_{1}+12(p+1)(p+3)\right\}\left(4-c_{1}^{2}\right) \delta^{2}\right] \\
= & F(c, \delta), \text { for } 0 \leq \delta=|x| \leq 1 .
\end{aligned}
$$

We assume that the upper bound for (3.27) occurs at an interior point of the set $\{(\delta, c): \delta \in[0,1]$ and $c \in[0,2]\}$. Differentiating $F(c, \delta)$ in (3.28) partially with respect to $\delta$, we get

$$
\begin{aligned}
& \frac{\partial F}{\partial \delta}=\left[2\left(p^{2}+4 p+7+6 p \cos \alpha\right) c^{2}\left(4-c^{2}\right)\right. \\
& \left.\quad+2\left\{\left(p^{2}+4 p+7\right) c^{2}-8(p+2)^{2} c_{1}+12(p+1)(p+3)\right\}\left(4-c^{2}\right) \delta\right] .
\end{aligned}
$$

For $0 \leq \delta \leq 1$, for fixed $c$ with $0 \leq c \leq 2$ and $\left(-\frac{\pi}{2 p} \leq \alpha \leq \frac{\pi}{2 p}\right)$, from (3.29), we observe that $\frac{\partial F}{\partial \delta}>0$. Therefore, $F(c, \delta)$ is an increasing function of $\mu$, which contradicts our assumption that the maximum value of it occurs at an interior point of the set $\{(\delta, c): \delta \in[0,1]$ and $c \in[0,2]\}$.

Further, for a fixed $c \in[0,2]$, we have

$$
\max _{0 \leq \delta \leq 1} F(c, \delta)=F(c, 1)=G(c) \text {, say. }
$$

From the relations (3.28) and (3.30), upon simplification, we obtain

$$
\begin{array}{r}
G(c)=\left[-2\left\{p^{2}+4 p+7+2\left(p^{2}+4 p+1\right) p^{2} \cos ^{2} \alpha\right\} c^{4}\right. \\
\left.+48(1+p \cos \alpha) c^{2}+48(p+1)(p+3)\right] .
\end{array}
$$




\section{International Journal of Science and Research (IJSR) \\ ISSN (Online): 2319-7064}

Index Copernicus Value (2013): 6.14 | Impact Factor (2015): 6.391

$$
\begin{gathered}
G^{\prime}(c)=-8\left\{p^{2}+4 p+7+2\left(p^{2}+4 p+1\right) p^{2} \cos ^{2} \alpha\right\} c^{3} \\
+96(1+p \cos \alpha) c . \\
G^{\prime \prime}(c)=-24\left\{p^{2}+4 p+7+2\left(p^{2}+4 p+1\right) p^{2} \cos ^{2} \alpha\right\} c^{2} \\
+96(1+p \cos \alpha) .
\end{gathered}
$$

The maximum or minimum value of $G(c)$ is obtained for the values of $G^{\prime}(c)=0$. From the expression (3.32), we get

$$
\begin{array}{r}
8\left\{p^{2}+4 p+7+2\left(p^{2}+4 p+1\right) p^{2} \cos ^{2} \alpha\right\} c^{3} \\
+96(1+p \cos \alpha) c=0 .
\end{array}
$$

We now discuss the following cases.

Case 1. If $c=0$, then from (3.33), we obtain

$$
G^{\prime \prime}(c)=96(1+p \cos \alpha)>0, \text { because }|\alpha| \leq \frac{\pi}{2 p} .
$$

Therefore, by the second derivative test, $G(c)$ has a minimum value at $c=0$, which is ruled out.

Case 2. If $c \neq 0$, then from (3.34), we obtain

$$
c^{2}=\frac{12(1+p \cos \alpha)}{p^{2}+4 p+7+2\left(p^{2}+4 p+1\right) p^{2} \cos ^{2} \alpha} \text {. }
$$

Using the value of $c^{2}$ given (3.35) in (3.33), after simplifying, we get

$$
G^{\prime \prime}(c)=-192(1+p \cos \alpha)<0 \text {, because }|\alpha| \leq \frac{\pi}{2 p} .
$$

From the second derivative test, $G(c)$ has a maximum value at $c$, where $c^{2}$ is given by (3.35). From the expression (3.31), we have $G$-maximum value at $c^{2}$, after simplifying, it is given by

$$
\max _{0 \leq c \leq 2} G(c)=\frac{\begin{array}{c}
288\left(1+2 p \cos \alpha+p^{2} \cos ^{2} \alpha\right)+48(p+1)(p+3) \\
+p^{2}+4 p+7+2\left(p^{2}+4 p+1\right) p^{2} \cos ^{2} \alpha
\end{array}}{p^{2}+4 p+7+2\left(p^{2}+4 p+1\right) p^{2} \cos ^{2} \alpha}
$$

Considering only the maximum value of $G(c)$ at $c$, where $c^{2}$ is given by (3.35). From the expressions (3.27) and (3.36), upon simplification, we obtain

$$
\begin{aligned}
& \left|d_{1} c_{1} c_{3}+d_{2} c_{1}^{2} c_{2}+d_{3} c_{2}^{2}+d_{4} c_{1}^{4}\right| \\
& \quad \begin{array}{r}
72\left(1+2 p \cos \alpha+p^{2} \cos ^{2} \alpha\right)+12(p+1)(p+3) \\
\leq \frac{+p^{2}+4 p+7+2\left(p^{2}+4 p+1\right) p^{2} \cos ^{2} \alpha}{p^{2}+4 p+7+2\left(p^{2}+4 p+1\right) p^{2} \cos ^{2} \alpha}
\end{array}
\end{aligned}
$$

From the expressions (3.18) and (3.37), after simplifying, we get

$$
\begin{aligned}
& \left|a_{p+1} a_{p+3}-a_{p+2}^{2}\right| \\
& \leq \frac{p^{4}\left\{\begin{array}{r}
6\left(1+2 p \cos \alpha+p^{2} \cos ^{2} \alpha\right)+(p+1)(p+3)\left(p^{2}+4 p+7\right. \\
\left.+2\left(p^{2}+4 p+1\right) p^{2} \cos ^{2} \alpha\right)
\end{array}\right\}}{(p+1)(p+2)^{2}(p+3)\left\{2\left(p^{2}+4 p+1\right)+\left(p^{2}+4 p+7\right) p^{2} \sec ^{2} \alpha\right\}}
\end{aligned}
$$

This completes the proof of the theorem 3.4.

Choosing $p=1$ in (3.38) we have the following

\section{Corollary 3.5.}

[37] If $f(z) \in \operatorname{CVSP}(\alpha)$, then

$$
\left|a_{2} a_{4}-a_{3}^{2}\right| \leq \frac{17\left(1+\cos ^{2} \alpha\right)+2 \cos \alpha}{144\left(1+\sec ^{2} \alpha\right)} .
$$

For the choice of $p=1$ and $\alpha=0$ in (3.38) we have the following

\section{Corollary 3.6.}

If $f(z) \in C V(\alpha)$, then

$$
\left|a_{2} a_{4}-a_{3}^{2}\right| \leq \frac{1}{8}
$$

This inequality is sharp and concides with that of Janteng, Halim and Darus [14].

\section{References}

[1] Abubaker and M. Darus, "Hankel Determinant for a class of analytic functions involving a generalized linear differential operator", Int. J. Pure Appl.Math. 69(4) (2011), 429-435.

[2] J.W. Alexander, "Functions which map the interior of the unit circle upon simple regions", Ann. of Math., 17 (1915), 12-22.

[3] R.M. Ali, "Coefficients of the inverse of strongly starlike functions", Bull. Malays. Math. Sci. Soc. (second series) 26(1) (2003), 63-71

[4] O. Al-Refai and M. Darus, "Second Hankel determinant for a class of analytic functions defined by a fractional operator", European J. Sci. Res. 28(2) (2009), 234-241.

[5] M. Arif, K.I. Noor and M. Raza, "Hankel determinant problem of a subclass of analytic functions", J. Ineqal. Appl., 2012 (2012), No 22, 7 pages.

[6] Deepak Bansal, "Upper bound of second Hankel determinant for a new class of analytic functions", Appl Math. Lett. 26 (2013), 103-107

[7] P.L. Duren, "Univalent functions", Vol. 259 of Grundlehren der Mathematischen Wissenschaften, Springer, New York, USA, (1983).

[8] R. Ehrenborg, "The Hankel determinant of exponential polynomials" Amer. Math. Monthly 107(6) (2000), $557-$ 560.

[9] A.W. Goodman, "Univalent functions" Vol.I and Vol.II Tampa, Florida: Mariner publishing Comp. Inc., 1983.

[10]U. Grenander and G. Szego, "Toeplitz forms and their application", Berkeley and Los Angeles: Univ. of California Press, 1958.

[11] T. Hayami and S. Owa, "Generalized Hankel determinant for certain classes", Int. J. Math. Anal., 4, No 49-52 (2010), 2573-2585.

[12]T. Hayami and S. Owa, "Applications of Hankel determinant for $\mathrm{p}$-valent starlike and convex functions of order $\alpha$ ", Far East J. Appl. Math., 46, No 1 (2010), 123.

[13] W.K. Hayman, "On the Second Hankel determinant of mean univalent functions", Prpc. Lond. Math. Soc., 3 (1968), 77-94.

[14] A. Janteng, S. A. Halim and M. Darus, "Hankel Determinant for starlike and convex functions", Int. J. Math. Anal. 1(13) (2007), 619-625.

[15]A. Janteng, S.A. Halim and M. Darus, "Coefficient inequality for a function whose derivative has a positive real part”, J. Inequal. Pure Appl. Math 7(2) (2006), 1-5.

[16] J. W. Layman, "The Hankel transform and some of its properties", J. Integer Seq. 4(1) (2001), 1-11.

[17] T.H. Mac Gregor, "Functions whose derivative have a positive real part", Trans. Amer. Math. Soc. 104(3) (1962), 532-537.

[18]A. K. Mishra and P. Gochhayat, "Second Hankel Determinant for a class of Analytic Functions Defined by Fractional Derivative", Int. J. Math. Math. Sci., vol 2008, Article ID 153280 (2008), 1-10. 
[19] N. Mohammed, D. Mohamed and S. Cik Soh, "Second Hankel determinant for certain generalized classes of analytic functions", Int. J. Math. Anal., 6, No 17-20 (2012), 807-812.

[20] Murugusundaramoorthy, Gangadharan., Magesh, N., "Coefficient inequalities for certain classes of analytic functions associated with Hankel determinant", Bull Math Anal. Appl. 1(3) (2009), 85-89.

[21] J. W. Noonan and D. K. Thomas, "On the second Hankel determinant of a really mean p-Valent functions", Trans. Amer. Math. Soc. 223(2) (1976), 337346.

[22] K. I. Noor, "Hankel determinant problem for the class of functions with bounded boundary rotation", Rev. Roum. Math. Pures Et Appl. 28(8) (1983), 731-739.

[23] S. Owa and H. M. Srivastava, "Univalent and starlike generalised hypergeometric functions", Canad. J. Math. 39(5) (1987), 1057-1077.

[24] Ch. Pommerenke, "Univalent functions", Gottingen: Vandenhoeck and Ruprecht, 1975.

[25] Ch. Pommerenke, "On the Hankel determinants of univalent functions”, Mathematika, 14 (1967), 108-112.

[26] Ch. Pommerenke, "On the coefficients and Hankel determinants of univalent functions", J. Lond. matha. Soc., 41 (1966), 111-122.

[27] T. RamReddy and D. Vamshee Krishna, “An upper bound to the second Hankel determinant for a subclass of analytic functions", Bull. Inst. Math. Virtual Inst., 4 (2014), 17-26.

[28] Sakaguchi, "On a certain univalent mapping”, J. Math. Soc. Japan, 11 (1959), 72-80.

[29] B. Simon, "Orthogonal polynomials on the unit circle", Part 1. Classical theory, American Mathematical Society Colloquium Publications, 54, Part 1, American Mathematical Society, Providence, RI, 2005.

[30]L. Spacek, "Contribution a la theorie des functions univalents (in Czech)”, Casop Pest. Mat. Fys., 62(1933), 12-19.

[31]D. Vamshee Krishna and T.RamReddy, "An upper bound to the second Hankel determinant for a subclass of analytic functions", Proc. Jangeon Math. Soc., 16, No 4 (2013), 559-568.

[32]D. Vamshee Krishna and T. RamReddy, "Coefficient inequality for uniformly convex functions of order $\alpha$ ", J. Adv. Res. Pure Math., 5, No 1 (2013), 25-41.

[33]D. Vamshee Krishna and T. RamReddy, "Coefficient inequality for certain subclasses of analytic functions", New Zealand J.Math., 42 (2012), 217-228.

[34]D. Vamshee Krishna and T. RamReddy, "Coefficient inequality for certain subclass of analytic functions", Armenian J. Math., 4, No 2 (2012), 98-105.

[35]D. Vamshee Krishna and T. RamReddy, "Hankel determinant for $\mathrm{p}$-valent starlike and convex functions of order $\alpha "$, Novi Sad J. Math., 42, No 2 (2012), 89-96.

[36]D. Vamshee Krishna and T. RamReddy, "An upper bound to the non-linear functional assocated with Hankel determinant for a subclass of analytic functions", Ind. J. Maths., 54, No 3 (2012), 343-355.

[37]D. Vamshee Krishna and T. RamReddy, "Coefficient Inequality for cetain subclasses of analytic functions associated with Hankel Determinant", Indian J. Pure Appl. Math., 46 (1) (2015): 91-106 